\section{Quantum oscillations between two weakly coupled reservoirs of superfluid ${ }^{3} \mathrm{He}$}

\author{
S. V. Pereverzev ${ }^{\star}$, A. Loshak, S. Backhaus, J. C. Davis \\ \& R. E. Packard
}

Physics Department, University of California, Berkeley, California 94720, USA

Arguments first proposed over thirty years ago, based on fundamental quantum-mechanical principles, led to the prediction ${ }^{1-3}$ that if macroscopic quantum systems are weakly coupled together, particle currents should oscillate between the two systems. The conditions for these quantum oscillations to occur are that the two systems must both have a well defined quantum phase, $\phi$, and a different average energy per particle, $\mu$ : the term 'weakly coupled' means that the wavefunctions describing the systems must overlap slightly. The frequency of the resulting oscillations is then given by $\boldsymbol{f}=\left(\mu_{2}-\mu_{1}\right) / \boldsymbol{h}$, where $\boldsymbol{h}$ is Planck's constant. To date, the only observed example of this phenomenon is the oscillation of electric current between two superconductors coupled by a Josephson tunnelling weak link ${ }^{4}$. Here we report the observation of oscillating mass currents between two reservoirs of superfluid ${ }^{3} \mathrm{He}$, the weak link being provided by an array of submicrometre apertures in a membrane separating the reservoirs. An applied pressure difference creates mass-current oscillations, which are detected as sound in a nearby microphone. The sound frequency (typically $6,000-200 \mathrm{~Hz}$ ) is precisely proportional to the applied pressure difference, in accordance with the above equation. These superfluid quantum oscillations were first detected while monitoring an amplified microphone signal with the human ear.

The theory underlying the above equation was developed in the context of generalizing the ideas of Josephson ${ }^{4}$. He predicted that in a superconducting tunnel junction, the quantum phase difference, $\Delta \phi$, across the function is related to the electrical current, $I$, through it, by the equation

$$
I=I_{\mathrm{c}} \sin (\Delta \phi)
$$

where $I_{\mathrm{c}}$ is the critical current of the junction. The phase difference evolves in time $t$ according to

$$
\frac{\partial(\Delta \phi)}{\partial t}=-\frac{\Delta \mu}{\hbar}
$$

where $\hbar$ is Planck's constant divided by $2 \pi$, and $\Delta \mu$ is the chemicalpotential $^{5}$ difference across the junction. For a fixed $\Delta \mu, \Delta \phi$ evolves in time as $\Delta \phi=-\Delta \mu t / \hbar$, and the corresponding current oscillates as $I=I_{\mathrm{c}} \sin (-\Delta \mu t / \bar{h})$. The chemical-potential difference (per Cooper pair) between two superconductors biased at voltage difference $\Delta V$ is given by $\Delta \mu=2 e \Delta V$ where $e$ is the charge on the electron, and the corresponding Josephson frequency $f_{\mathrm{j}}=2 e \Delta V / h=4.82 \times 10^{14} \mathrm{~Hz} \mathrm{~V}^{-1}$.

Equations (1) and (2), often called the d.c. and a.c. Josephson relations respectively, were re-derived in a more general context by Josephson, Anderson and Feynman ${ }^{1-3}$ who realized that the equations were applicable for any two, weakly coupled, phase-coherent systems. The dramatic aspect of the superconducting case is that the two coupled systems are macroscopic rather than microscopic.

For several decades physicists have recognized a close analogy between superconductors and superfluid helium ${ }^{6}$ in that they are both systems where a large number of particles occupy a single coherent quantum state. It has therefore been natural to search for quantum current oscillations in superfluids. The required condi-

* Permanent address: Institute for High Pressure Physics, Russian Academy of Sciences, Russia. tions for the oscillations to exist in superfluids are that two samples of superfluid be separated by a region small enough so that the wavefunctions of each part can weakly penetrate and overlap that of the other. An example of this would be two reservoirs of superfluid helium in contact through a small aperture. Both the diameter and the length of the aperture must be comparable to the superfluid healing length ${ }^{7}$. This is the minimum length scale over which the magnitude of the superfluid wave function is allowed to vary for
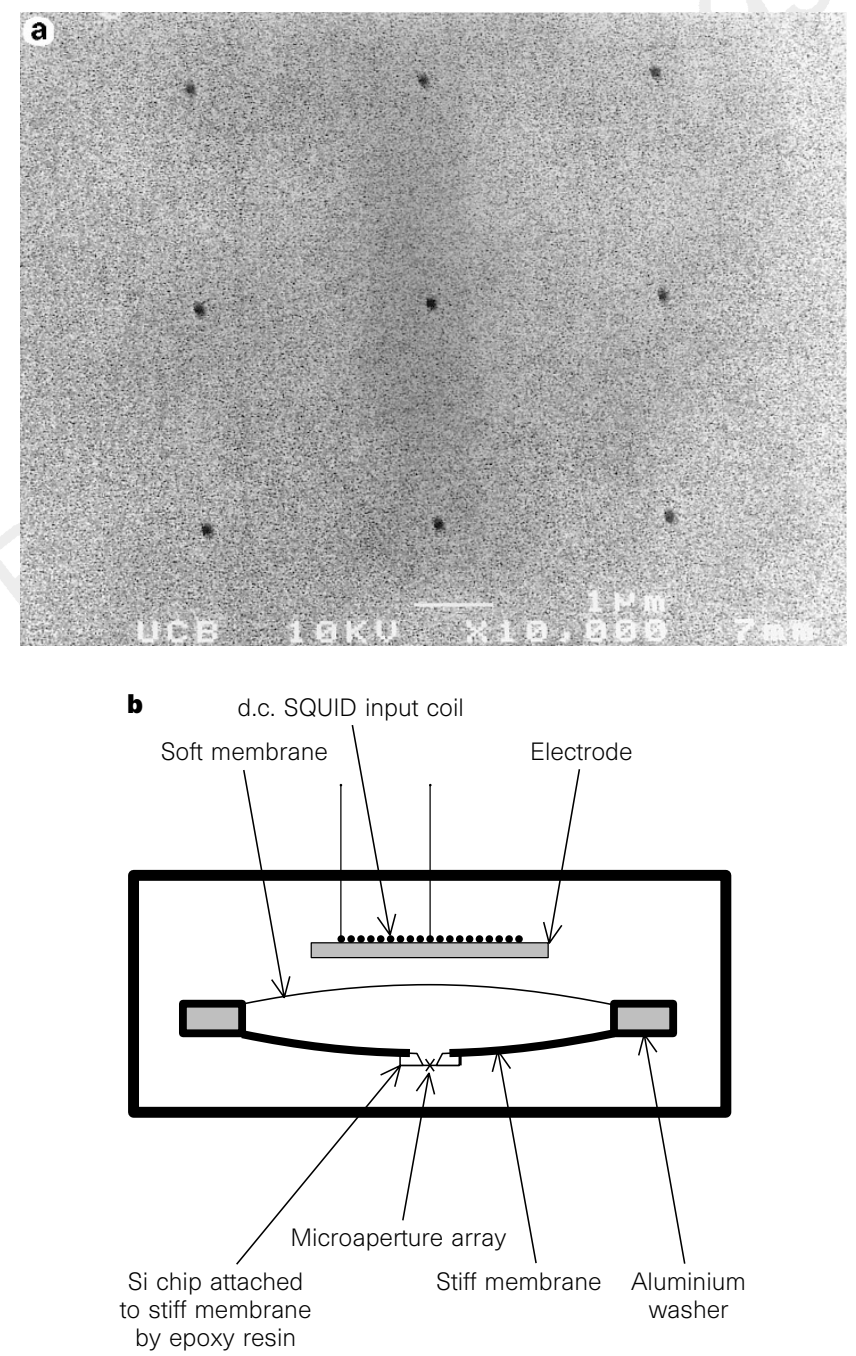

Figure $1 \mathbf{a}$, Scanning electron micrograph of a small area of the array of apertures in a silicon nitride ( $\mathrm{SiN}$ ) membrane whose thickness is $50 \mathrm{~nm}$. It shows the $3-\mu \mathrm{m}$ separation between each aperture, and that the aperture diameter is near $100 \mathrm{~nm}$. Larger-area scans showing the whole array confirm that there are 4,225 holes. Scale bar at bottom, $1 \mu \mathrm{m}$. b. Schematic diagram of the experimental cell. The pillbox-shaped inner cell consists of an 140- $\mu$ m-thick aluminium washer, to which are attached (by epoxy resin) a stiff diaphragm on the lower side, and a very soft $\left(1140 \pm 170 \mathrm{Nm}^{-1}\right)$ diaphragm on the top. The Si chip, containing the SiN membrane through which the array of apertures has been etched, is glued in position (by epoxy resin) over a small hole in the lower membrane. The upper membrane is metallized on the outside by evaporating a 100-nm layer of $\mathrm{Pb}$, and then a $20-n m$ layer of In, onto its surface. It is at the same potential as the Al washer. About $100 \mu \mathrm{m}$ above the upper membrane is a thin metallized electrode. Changes in the potential of this electrode relative to the membrane are used to apply forces to the soft membrane. Approximately $10 \mu \mathrm{m}$ behind this electrode are the superconducting wires which form the input coil of the d.c. SQUID. This system is used as the sensitive position sensor. This whole assembly is immersed in a container of superfluid ${ }^{3} \mathrm{He}$ in contact with a nuclear demagnetization refrigerator. 
reasons of energy minimization. We refer to such an aperture as a weak link.

The equations which are the superfluid equivalent of the superconducting Josephson equations, have the same form as equations (1) and (2). The chemical-potential difference per particle on the right side of equation (2) becomes $\Delta P m / \rho$. Here $\Delta P$ is the pressure difference across the weak link, $\rho$ is the liquid density, and $m$ is the mass of either the ${ }^{4} \mathrm{He}$ atom or twice the ${ }^{3} \mathrm{He}$ atomic mass, depending on whether one is describing ${ }^{4} \mathrm{He}$ or ${ }^{3} \mathrm{He}$. (We are neglecting a small temperature term in the chemical potential because it is almost negligible in the experiments reported here.) In equation (1) the current, $I$, now refers to a mass current rather than an electric current. The pressure difference should therefore be accompanied by a mass supercurrent oscillating at the frequency

$$
f=\frac{\Delta P m}{\rho h}
$$

which is $69 \mathrm{kHz} \mathrm{Pa}^{-1}$ for ${ }^{4} \mathrm{He}$, and $183.7 \mathrm{kHz} \mathrm{Pa}^{-1}$ for ${ }^{3} \mathrm{He}$. We emphasize that this effect does not appear in ordinary fluids, or even for superfluids flowing through tubes which are not weak links. In the weak link, the fluid's response to an applied pressure difference is not simply to accelerate in proportion to that pressure gradient, but rather to oscillate in place at a frequency which is proportional to the pressure difference.

Demonstration of superfluid effects relying on these equations has been very elusive ${ }^{8}$. The fundamental problem is that a superfluid weak link requires an aperture whose dimensions are on the scale of the superfluid healing length, which is only $\sim 0.1 \mathrm{~nm}$ for ${ }^{4} \mathrm{He}$ and $\sim 50 \mathrm{~nm}$ for ${ }^{3} \mathrm{He}$. To observe quantum oscillation phenomena one

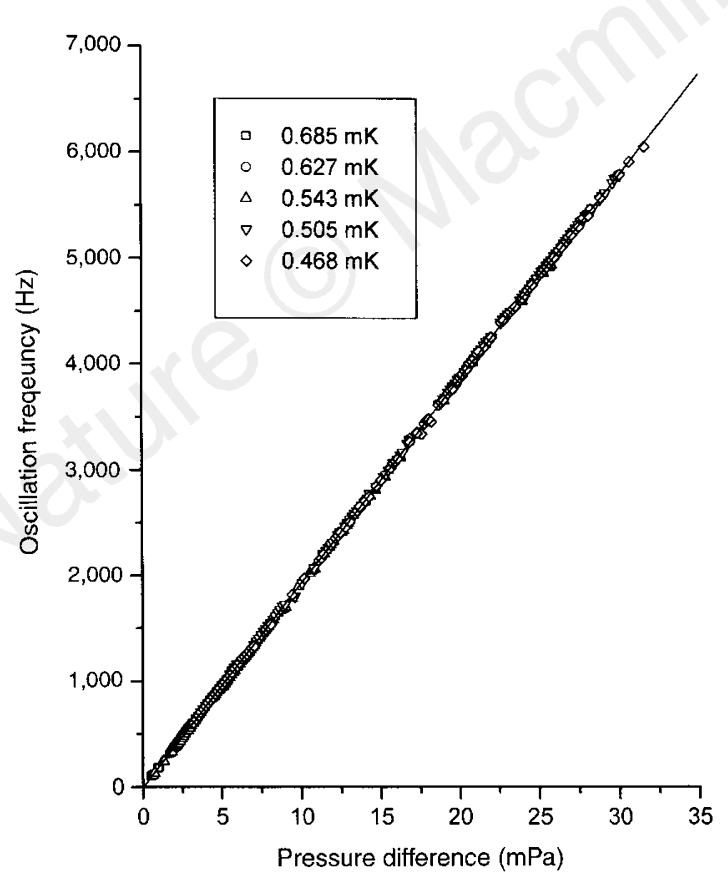

Figure $2 \mathrm{~A}$ graph of the frequency of the quantum oscillation detected at the top membrane as a function of $\Delta P$, the pressure difference across the weak-link array. Data for five different temperature $T(0.468,0.505,0.543,0.627,0.685 \mathrm{mK})$ are plotted. A line is fitted to all the data points, as shown. The slope of the line is $194 \mathrm{kHz} \mathrm{Pa}^{-1}$ with a statistical error of $\pm 1 \mathrm{kHz} \mathrm{Pa}^{-1}$, and a systematic uncertainty of $\pm 15 \mathrm{kHzPa}^{-1}$. The larger systematic error comes from uncertainties in the capacitance between the soft membrane and the electrode above it. The excellent linear relationship between $f$ and $\Delta P$, and the measured value of the slope (which is predicted to be $183.7 \mathrm{kHz} \mathrm{Pa}^{-1}$ ), provide a very clear confirmation of equation (3) over more than two orders of magnitude of pressure. For the data at each individual temperature, the $f$ versus $\Delta P$ slope has been measured, and the differences between these measured slopes are $<0.5 \%$ must first solve two technical problems; the fabrication of such small structures, and the measurement of the extremely small mass currents that would flow through such an aperture. Although these problems are not yet completely solved for ${ }^{4} \mathrm{He}$ (ref. 9), recent advances have succeeded in both goals for ${ }^{3} \mathrm{He}$. Micro-fabrication techniques have led to the development of apertures which are submicrometre in all three dimensions ${ }^{10}$. At the same time, displacement transducer development ${ }^{11}$ (for gravity-wave detection) has led to technology that enables one to monitor the motion of a fluid-driving piston ${ }^{12}$, with sensitivity sufficient to detect the small currents predicted ${ }^{13-18}$ in a superfluid weak link. These technical advances have set the stage for observation of Josephson effects in ${ }^{3} \mathrm{He}$, and for study of the rich physical phenomena analogous to those observed in superconductors.

There is already one positive experimental indication ${ }^{19,20}$ of a ${ }^{3} \mathrm{He}$ superfluid current-phase relation similar to equation (1). In that work, the superfluid ${ }^{3} \mathrm{He}$ mass flow was through a single submicrometre aperture connected in parallel with a larger tube. The authors of references 19 and 20 report that, over a limited temperature range, they can numerically simulate the observed response of their nonlinear superfluid ${ }^{3} \mathrm{He}$ oscillator, by using equations of motion that incorporate equations (1) and (2) into the dynamics of the system.

Encouraged by these reports, we have pursued the development of a ${ }^{3} \mathrm{He}$ weak link ${ }^{21,22}$. Our device uses a square array (Fig. 1a) of 4,225 apertures spaced by $3,000 \mathrm{~nm}$. Each aperture is $100 \mathrm{~nm}$ in diameter, in the same 50 -nm-thick, silicon nitride membrane ${ }^{10}$. We use this ${ }^{3} \mathrm{He}$ weak link in a temperature range where the superfluid healing length is greater than, or at least comparable to, the aperture diameter. We designed the array with the hope that the apertures would act coherently and have no interference between adjacent apertures, so that the array should behave like a single aperture but with 4,225 times greater current. One of the significant discoveries implied by the results reported below is the fact that the array does indeed behave in this desired manner.

The silicon chip containing the aperture array is placed in the wall of a pill-box-shaped cell, whose top and bottom surfaces are plastic membranes (Fig. 1b). The array is located in a lower, stiff membrane and the position of the upper, soft membrane is monitored with a sensitive displacement detector which can resolve motion as small as $10^{-14} \mathrm{~m} \mathrm{~Hz}^{-1 / 2}$. The upper membrane can be manipulated by the application of bias voltages between its conducting surface and a fixed electrode. The cell has an internal volume of $\sim 10 \mathrm{~mm}^{3}$, and takes several hours to fill by flow of normal liquid ${ }^{3} \mathrm{He}$ through the array at temperature near $1 \mathrm{~K}$. This cell is placed in a ${ }^{3} \mathrm{He}$-filled enclosure, which is in contact with a nuclear demagnetization refrigerator capable of cooling the ${ }^{3} \mathrm{He}$ to temperatures well below its superfluid transition temperature $\left(T_{\mathrm{c}}=0.929 \mathrm{mK}\right)$.

We apply a step increase in bias potential to the soft membrane, which pulls it abruptly toward the fixed electrode. This creates a stepwise change in the pressure difference $\Delta P$ across the array, from zero initial pressure to a value determined by the applied voltage. We record the subsequent motion of the membrane's position, $x(t)$, as mass flow through the array allows it to relax towards its new equilibrium position $x_{\mathrm{f}}$, where $\Delta P$ is again zero. The value of $\Delta x \equiv x_{\mathrm{f}}-x(t)$ is a measure of the instantaneous pressure across the aperture. If the aperture array behaves like a quantum weak link, the relaxing pressure difference $\Delta P(t)$ should be accompanied by mass-current oscillations within the aperture whose frequency is determined by equation (3).

A typical flow transient shows a relaxation of $x(t)$ towards equilibrium. Owing to vibration noise in the displacement transducer, an oscilloscope trace (of the instantaneous voltage which is proportional to $x$ ) exhibits no remarkable structure suggestive of the predicted quantum oscillations. But if the electrical output of the displacement transducer is amplified and connected to audio headphones, the listener makes a most remarkable observation. As the 
pressure across the array relaxes to zero there is a clearly distinguishable tone smoothly drifting from high to low frequency during the transient, which lasts for several seconds. This simple observation marks the discovery of coherent quantum oscillations between weakly coupled superfluids.

To quantify this assertion we have processed several digitally recorded transient signals. Each transient is broken into equal, short time intervals. The average membrane position during the interval is used to calculate the pressure difference. For each time interval we compute the power spectrum of the membrane motion. As the frequency is drifting during any interval, the peak in the Fourier transform is not sharp. We identify the centre frequency of the strongest spectral component in the range of interest. This is the oscillation frequency corresponding to the pressure difference across the weak link during that interval.

Figure 2 plots the oscillation frequency, $f$, as a function of $\Delta P$ for a series of different temperatures. The frequency is found to be proportional to $\Delta P$, the proportionality factor being $194 \mathrm{kHz} \mathrm{Pa}^{-1}$, with a statistical error of $\pm 1 \mathrm{kHz} \mathrm{Pa}^{-1}$, and a systematic uncertainty of $\pm 15 \mathrm{kHz} \mathrm{Pa}^{-1}$. The linear relationship between $f$ and $\Delta P$, and the measured value of the slope, are in clear agreement with equation (3), over a pressure range of more than two orders of magnitude. This is a direct confirmation of the quantum-oscillation equation for superfluid ${ }^{3} \mathrm{He}$.

The relation between frequency and pressure difference is observed to be independent of temperature, to within the error of the measurement. It is expected ${ }^{13,14,17}$ that the actual current-phase relation might become increasingly distorted from the simple sine form in equation (1) as the coherence length gets smaller with falling temperature ${ }^{7}$. The distorted $I(\Delta \phi)$ would lead to higher harmonics of the quantum oscillations. We have searched for these higher harmonics in the spectral signal of the sound, and find that (to within our signal-to-noise ratio) they are absent over a wide range of temperatures.

The experimental results reported here lead to three significant conclusions. (1) The aperture array behaves like a single coherent superfluid weak link. (2) The current-phase relation is similar to the d.c. Josephson equation (equation (1)), that is periodic with modulus $2 \pi$. (3) The time evolution of the quantum phase across the array leads to current oscillations that drive the upper diaphragm at the frequency predicted by equation (3).

The remarkable properties of the weak-link array may lead to the observation of several physical phenomena analogous to those found in superconductivity. These include Shapiro ${ }^{23}$ steps and, perhaps most significantly, the development of the superfluid analogue of a d.c. SQUID ${ }^{24}$.

\section{Received 5 June; accepted 30 June 1997.}

1. Josephson, B. D. Possible new effects in superconductive tunneling. Thesis, Cambridge Univ. (1962) 2. Anderson, P. W. in Lectures on the Many-Body Problem (ed. Caianello, E. R.) 113-135 (Academic, New York, 1964).

3. Feynman, R. P. The Feynman Lectures on Physics Sect. 21-9 (Addison Wesley, New York, 1965)

4. Josephson, B. D. Possible new effects in superconductive tunnelling. Phys. Lett. 1, 251-253 (1962).

5. Reif, F. Fundamentals of Statistical and Thermal Physics Sect. 8-7 (McGraw-Hill, New York, 1965).

6. Tilley, D. R. \& Tilley, J. Superfluidity and Superconductivity (Hilger, New York, 1990).

7. Vollhardt, D. \& Woolfle, P. The Superfluid Phases of Helium-3 Sect. 7-2 (Taylor \& Francis, New York, 1990)

8. Tilley, D. R. \& Tilley, J. Superfluidity and Superconductivity Sect. 7-6 (Hilger, New York, 1990).

9. Pereverzev, S. V. Toward the Josephson effect in superfluid helium: how to make $20 \mathrm{~nm}$ holes in a $10 \mathrm{~nm}$ thick membrane. J. Low Temp. Phys. 101, 573-579 (1995).

10. Amar, A., Sasaki, Y., Lozes, R. L., Davis, J. C. \& Packard, R. E. Fabrication of submicron apertures in thin membranes of silicon nitride. J. Vac. Sci. Technol. B 11, 259-262 (1993).

11. Paik, H. J. Superconducting tunable-diaphragm transducer for sensitive acceleration measurements $J$. Appl. Phys. 47, 1168-1178 (1976)

12. Avenel, O. \& Varoquaux, E. in Proc. 10th Int. Cryogenic Eng. Conf. (eds Klipping, G. \& Klipping, I.) 587-589 (Butterworths, Guildford, 1986).

13. Monien, H. \& Tewordt, L. Theory of Josephson flow oscillations in superfluid ${ }^{3} \mathrm{He}-\mathrm{B}$. J. Low Temp. Phys. 62, 277-300 (1986).

14. Hook, J. R. Current-phase relation for flow of an $s$-wave paired superfluid through a cylindrical channel of finite length. Jpn. J. Appl. Phys. 26, 159-160 (1987).

15. Kurkijärvi, J. Josephson current through a short and narrow orifice in a p-wave superfluid. Phys. Rev. B 38, 11184-11187 (1988).

16. Thuneberg, E. V. Two dimensional simulation of the Josephson Effect in superfluid ${ }^{3} \mathrm{He}$. Europhys. Lett. 7, 441-446 (1988)

17. Ullah, S. \& Fetter, A. L. Superfluid ${ }^{3} \mathrm{He}-\mathrm{B}$ in a weak link. Phys. Rev. B 39, 4186-4190 (1989).
18. Thuneberg, E. V., Kurkijärvi, J. \& Sauls, J. A. Quasiclassical theory of the Josephson effect in superfluid ${ }^{3}$ He. Physica B 165\&166, 755-756 (1990).

19. Avenel, O. \& Varoquaux, E. Josephson effect and quantum phase slippage in superfluids. Phys. Rev. Lett. 60, 416-419 (1988).

20. Varoquaux, E., Avenel, O., Ihas, G. \& Salmelin, R. Phase slippage in superfluid ${ }^{3} \mathrm{He}-$ B. Physica B 178, 309-316 (1990).

21. Steinhauer, J. The current-pressure relation for a superfluid ${ }^{3} \mathrm{He}$ weak link. Thesis, Univ. California, Berkeley (1995)

22. Mukharsky, Yu. M., Loshak, A., Schwab, K., Davis, J. C. \& Packard, R. E. Study of an array of superfluid ${ }^{3}$ He weak links. Czech. J. Phys. 46, 115-116 (1996).

23. Shapiro, S. Josephson currents in superconducting tunnelling; the effect of microwaves and other observations. Phys. Rev. Lett. 11, 80-82 (1963).

24. Packard, R. E. \& Vitale, S. Principles of superfluid-helium gyroscopes. Phys. Rev. B 46, 3540-3549 (1992).

Acknowledgements. In early stages of this work we were helped by J. Steinhauer, K. Schwab, Yu. M. Murkarsky and I. Schuster. E. W. Hudson made important contributions to the data acquisition system. Figure $1 \mathrm{~b}$ was made by R. Orr. The aperture array was constructed at the Berkeley Microfabrication Laboratory. This work was supported in part by the National Science Foundation, the Office of Naval Research, and the Packard Foundation.

Correspondence and requests for materials should be addressed to R.E.P. (e-mail: packard@socrates. berkeley.edu)

\section{Graphitic cones and the nucleation of curved carbon surfaces}

\section{A. Krishnan*, E. Dujardin †, M. M. J. Treacy ${ }^{\star}$, J. Hugdahl $\ddagger$, S. Lynum $¥ \&$ T. W. Ebbesen $* \$$}

* NEC Research Institute, 4 Independence Way, Princeton, New Jersey 08540, USA $\dagger$ Laboratoire de Chimie, Collège de France, 11, place M. Berthelot, 75005 Paris, France

$\ddagger$ Kvaerner Engineering a.s., 1324 Lysaker, Norway

$\$$ ISIS, Louis Pasteur University, rue Blaise Pascal, 67000 Strasbourg, France

The nucleation and growth of curved carbon structures, such as fullerenes, nanotubes and soot, are still not well understood. A variety of models have been proposed ${ }^{1-17}$, and it seems clear that the occurrence of pentagons, which yield $60^{\circ}$ disclination defects in the hexagonal graphitic network, is a key element in the puzzle. The problem of nucleation has been complicated by the great variety of structures observed in any one sample. Here we report an unusual carbon sample generated by pyrolysis of hydrocarbons, consisting entirely of graphitic microstructures with total disclinations that are multiples of $+60^{\circ}$. The disclination of each structure corresponds to the presence of a given number of pentagons in the seed from which it grew: disks (no pentagons), five types of cones (one to five pentagons), of which only one was known previously ${ }^{18}$, and open tubes (six pentagons). Statistical analysis of these domains shows some unexpected features, which suggest that entropy plays a dominant role in the formation of disclinations. Furthermore, the total disclination of a domain is determined mainly at the nucleation stage.

Several models have been proposed for the formation of fullerenes, which are also relevant to the nucleation of other curved structures such as nanotubes. The "pentagon-road"1,3 and the "ringstacking" models ${ }^{4}$ are among the best known. The essence of the pentagon-road model is that small flat hexagonal networks of carbon have so much dangling-bond energy at the edges that, if the temperature and timescale are such as to permit annealing, the structures will try to eliminate dangling bonds by folding and incorporating pentagons until the structure is closed (12 pentagons or $4 \pi$ total disclination). Dynamic calculations support this idea ${ }^{5}$. Reduction of the reaction enthalpy $\Delta H$ is the overriding concern in the pentagon-road model. In the ring-stacking model, it is proposed that curved surfaces are created by the selective assembly of monocyclic carbon rings ${ }^{4}$, to account for the fact that only very few of the possible fullerene isomers are formed ${ }^{15}$. As it considers only certain reaction paths, the ring-stacking model is essentially driven by entropy changes $(\Delta S)$. Monocyclic rings are known to be the 